\title{
Lob des Kleinglaubens oder Humor im Dienst des Zweifelns
}

Einem Mann zum 90. Geburtstag gewidmet, dem »der Zweifel so teuer wie der Glaube« war ${ }^{1}$

\section{Zweifel als Bedrohung}

Aus der Sicht vieler Gläubigen wird Zweifel vornehmlich negativ wahrgenommen: Nach ihrer Meinung stellt er eine Gefahr, eine Bedrohung für den Glauben dar. Will der Glaube Gewissheit stiften, so provoziert Zweifel Beunruhigung, die diese Gewissheit mehr oder weniger stark erschüttert. Während der Glaube Einklang, Eintracht mit sich selbst schenken, dem Gläubigen ein Bei-sich-Sein ermöglichen will, lässt der Zweifel den störenden Aspekt einer Zweiheit aufkommen, die Zweiklang, Zwietracht, ja sogar Zwiespalt auslöst ${ }^{2}$. Anstatt in Frieden bei sich zu sein, schwankt dann der Mensch hin und her. So formuliert es der Jakobusbrief in aller Schärfe: "wer zweifelt, gleicht den Wogen des Meeres, die vom Wind gepeitscht und dahin und dorthin geschlagen werden" $(1,6)$. Deshalb gilt es im Glauben zu bitten, ohne zu zweifeln (V. 6a). Und so gilt denn für den Judasbrief Zweifeln als etwas Erbarmungswürdiges: "Erbarmt euch derer, die zweifeln!« (Judas 22)

Auch in den griechischen Vokabeln, die das Neue Testament für unser Thema braucht, kommt der soeben betonte Aspekt der Zweiheit, des Zwiespalts, zum Ausdruck: sie sind mit den Präfixen dia oder dis gebildet. Wenn ich richtig sehe, kennt das Neue Testament Zweifel als Substantiv kaum: distagmos kommt nicht vor; höchstens könnte dialogismos manchmal Gedanken im Sinne von Zweifelsgedanken, von Bedenken, meinen (so etwa in Lukas 24,38, bei den Jüngern dem Auferstandenen gegenüber). Wohl aber gibt es an mehreren

\footnotetext{
1 F. Dürrenmatt, Turmbau, Zürich 1990, 231f.

2 Etymologisch scheint Zweifel von "zwei« und "-falt« zu kommen und deshalb etwas "Zweifältiges, Gespaltenes« zu meinen (vgl. Friedrich Kluge, Etymologisches Wörterbuch der deutschen Sprache, Berlin/New York, $\left.{ }^{22} 1989,820\right)$.
} 
Stellen das Zweifeln als nomen actionis, mit den Verben distazein, mehr als einem aktiven Vollzug, und diakrinesthai, mehr als einem passiven Widerfahrnis (von Zweifeln, von Bedenken befallen werden).

Weil im Zweifeln also das Hin- und Hergetriebenwerden akzentuiert wird, das die Gewissheit aus der Ruhe reisst, wird im Neuen Testament vor allem vor dem Zweifel gewarnt, dazu aufgerufen, gegen ihn anzukämpfen, zu glauben ohne zu zweifeln (z. B. in Markus 11,23, in Hinsicht darauf, was durch Beten erreicht werden kann). Und so heisst es denn auch von Abraham bei Paulus (Röm 4,20), er habe sich nicht durch Unglauben in Zweifel an Gottes Verheissung versetzen lassen, sondern sei stark im Glauben geworden.

\section{Humor als Arbeit an der Negativität des Zweifelns}

Muss es dabei bleiben, dass dem Glauben das Zweifeln als Gefahr gegenüber steht? Ist diese negative Sicht die einzig mögliche, oder könnte die Beziehung zwischen Glauben und Zweifeln auch eine konstruktive werden?

Ich möchte diese Fragen auf einem Umweg behandeln, indem ich mit Hilfe des Humors die Negativität hinterfrage, die man dem Zweifeln zuspricht. Das sei hier durch drei Zeichnungen eines französischen Zeichners namens Piem ${ }^{3}$ unternommen, die alle drei dazu einladen, sich lachend mit der Spannung von Glauben und Zweifeln auseinanderzusetzen.

3 Piem, Dieu et vous, Paris 1996. 


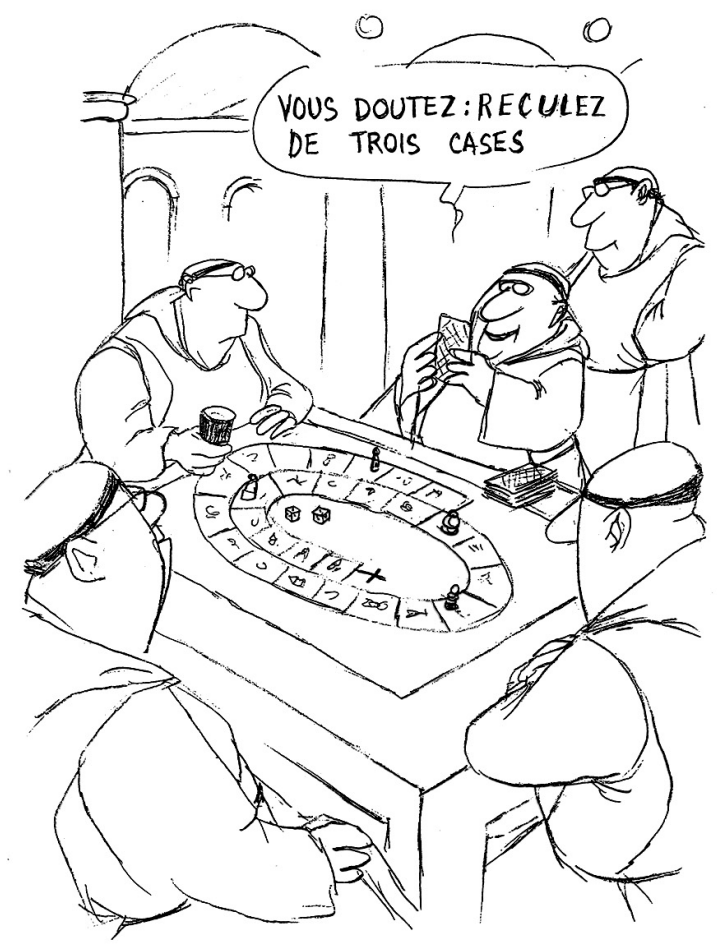

Noch gilt in dieser ersten Zeichnung der Zweifel nicht ganz ernst. Im Kloster scheint es, so die implizite Annahme des Zeichners, das Zweifeln nur im Spiel zu geben. Diese gemütlich spielenden Mönche scheinen auch nicht besonders dem Zweifel ausgesetzt zu sein. Nur etwas zufällig kann man beim Gänsespiel auf das falsche Feld geraten, und dann heisst in der betreffenden Spielanweisung: "Sie zweifeln: gehen Sie drei Felder zurück«. Der Zweifel ist also durchaus negativ konnotiert: Obschon alles im Spiel stattfindet, wird man bestraft. Drei Felder zurück, und deshalb umso länger unterwegs zum Ziel. Doch auch im Spiel gilt Ernst, und dieser könnte sich potentiell auch als Ernst im Leben erweisen: Auch im Leben könnte man wegen Zweifel zurückversetzt werden ... 

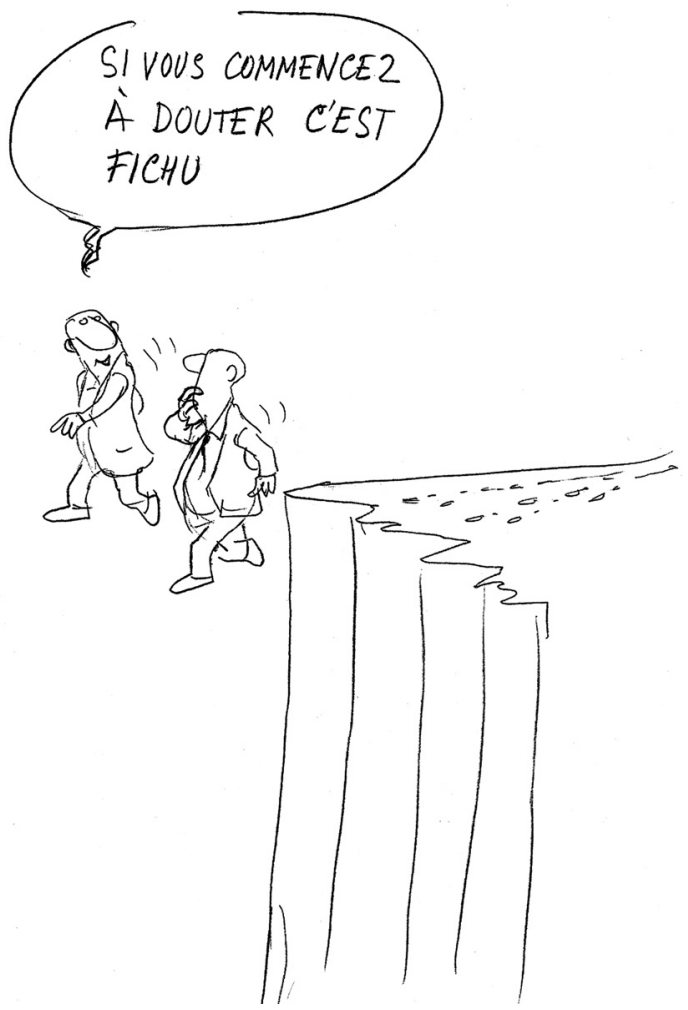

In dieser zweiten Zeichnung gilt es bereits viel ernster:Im Glauben hat man sich hinausgewagt, über den Abgrund hinweg, in der Annahme, alles sei möglich dem, der glaubt. Diese absolute Glaubensgewissheit trägt anscheinend. Doch könnte sich Zweifel einstellen: "Wenn Sie anfangen zu zweifeln, ist es im Eimer«. Diese Bemerkung, die beim Mitwanderer anscheinend etwas Verwirrung stiftet, zeigt, dass auch der absoluteste Glaube um diese Möglichkeit weiss. Indem sie hier erwähnt wird, wird sie auch hervorgerufen.Verstanden wird sie hier als die Gefahr eines Absturzes aus dem Wunder heraus in den drohenden Abgrund. Deshalb die Mahnung, ja nicht damit anzufangen. Aber hat man diesen Anfang nicht gerade dadurch schon ermöglicht, dass man dessen Möglichkeit erwogen hat? 


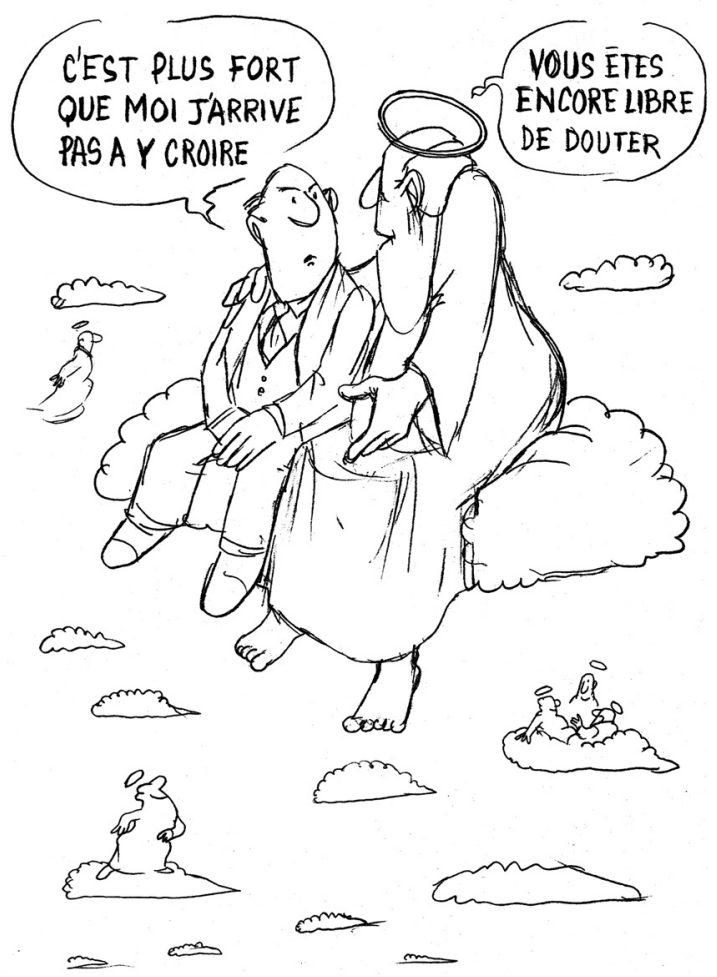

In dieser dritten Zeichnung liegt den zwei anderen gegenüber eine Steigerung der Spannung. Der Mensch sitzt nun gemeinsam mit Gott auf einer Wolke. Menschlich gesehen eine eher prekäre Situation, in der die Möglichkeit des Zweifelns nicht ohne Gefahr ist. Doch der Mensch spricht sein Nicht-glauben-Können aus: »Es ist stärker als ich, ich kann es nicht glauben«. Gott antwortet darauf mit der Möglichkeit des Zweifelns: "Sie sind noch frei zu zweifeln«. Bei Gott aufgehoben wähnt man sich im Sicheren, und doch ist es gerade Gott selbst, der die Freiheit zum Zweifeln zuspielt. Freilich nicht ohne gefährliche Konsequenzen, wie die Geste der göttlichen Hand anzeigt, die in den offenen Raum weist ... Wäre Gott etwa weniger absolut als die, welche zweifelsfrei an ihn glauben? 
Drei Situationen des Zweifels, in denen zum Lachen eingeladen wird. Doch nicht über den Zweifel soll gelacht werden, sondern vielmehr über den Glauben, der den Zweifel ausschalten muss. Gegen diesen Glauben wird das Zweifeln gerade stark gemacht. Der Humor lacht hier zugunsten des Zweifelns, und damit kommt indirekt, implizit, die Möglichkeit auf, dem Zweifeln eine grössere Positivität zuzusprechen.

\section{Der Zweifel wird im Kleinglauben aufgehoben}

»Humor enthält eine weit tiefere Skepsis als Ironie [...]«, hat einmal Kierkegaard gesagt: Während diese sich mit der Unwissenheit auseinandersetzt, verhalte sich der Humor "zu dem alten Satz: credo quia absurdum «; dadurch aber sei im Humor auch »eine weit tiefere Positivität«, denn »er bewegt sich nicht in humanen, sondern in gottmenschlichen Bestimmungen ${ }^{4}{ }^{4}$

Genau an dieser Stelle stehen wir, an der Negativität in Positivität umschlagen kann. Gewiss ist die Negativität nicht einfach überholt, denn damit wären wir wieder beim Glauben, der sich absolut setzt, der im Wahn lebt, zweifellos alles zu können. Die Positivität liegt vielmehr darin, dass es im Zeichen des credo quia absurdum dem Glauben gerade zu eigen ist, in einem ständigen Dennoch, in einem ständigen Trotzdem zu leben. Anders gesagt: Zweifeln gehört zum Glaubensvollzug, lässt diesen wachsen, reifen. Dadurch, dass er immer tiefer um die Möglichkeit des Zweifels in sich selbst weiss, wird er immer tieferer Glaube.

Das befreit den Glauben zu einer immer klareren Sicht über sich selbst, aber auch über den Menschen zwischen Gott und Welt. Nicht von ungefähr liegt im diakrinesthai neben dem Moment des Zweifelns auch das Moment des Unterscheidens und Urteilens. Zweifel ist demnach Bedingung des Unterscheidungs- und Urteilsvermögens, und dieses gehört wesenhaft zum reifen Glauben.

Im Matthäusevangelium scheint mir die Möglichkeit gegeben, dem Zweifel trotz aller Negativität diese positive Bedeutung zuzuschreiben. Das geschieht im Zeichen des Kleinglaubens (oligapistia). "Ihr Kleingläubige, Du Kleingläubiger!«, so spricht Jesus öfters seine

4 Über den Begriff der Ironie mit ständiger Rücksicht auf Sokrates, in : S. Kierkegaard, Gesammelte Schriften, Düsseldorf/Köln, 31. Abteilung, 1961, 334f. 
Jünger an. Nicht scharf verurteilend, sondern eher mit Sympathie sanft mahnend. Jüngerschaft als Nachfolge ist Kleinglaube. Die Chance des Kleinglaubens ist, dass er noch am Wachsen, am Reifen ist. Das kann gerade durch den Zweifel geschehen. So etwa geschieht es in Matthäus 14: Als der auf dem Wasser wandelnde Petrus plötzlich einsinkt und um Rettung schreit, fängt ihn Jesus auf. "Sogleich streckte Jesus seine Hand aus, hielt ihn fest«. Dabei sagt er ihm, Kleinglaube und Zweifel miteinander verknüpfend: »Du Kleingläubiger! Warum hast du gezweifelt?« (V. 31).

So darf der Zweifel auch zum grossen Finale des Evangeliums gehören. Beim Erscheinen des Auferstandenen werfen sich die Jünger nieder, verbeugen sich. Doch heisst es zugleich: »aber einige zweifelten « (Matthäus 28,17). Aber diese Zweifelnden dürfen dabei bleiben. Auch ihnen gilt die Verheissung: »Und seid gewiss: Ich bin bei euch alle Tage bis an der Welt Ende» (Matthäus 28,20).

Zweifeln ist im Kleinglauben aufgehoben, nicht im Sinne von »überholt, überwunden", sondern im Sinne von "aufgenommen, integriert«.

\section{Schwache Gewissheit, oder: von der Zerbrechlich- keit des Glaubens}

In recht ähnlicher Weise hat das Markusevangelium die Spannung von glauben und zweifeln mit der Figur des Vaters in Markus 9 erzählerisch inszeniert, als ausgeschriene Spannung von pisteuein und apistia. Als Jesus zu ihm sagt: "Was soll das heissen: Wenn du etwas vermagst? Alles ist möglich dem, der glaubt.«(V. 23), schreit der Vater des Kindes sogleich: "Ich glaube! Hilf meinem Unglauben!» (V. 24). Glaubensgewissheit ist keine absolute, Zweifel, Unglauben ausschaltende Gewissheit. Ist das Gebet des Vaters eine Art nacktes Glaubensbekenntnis, so ist es zugleich ein Bekenntnis zum Unglauben, der in diesem Glauben wohnt.

Dem entspricht, was Dürrenmatt in seinem letzten Buch Turmbau zum Ausdruck brachte:»Bekennen kann einer nur seinen Unglauben an etwas, seinen Zweifel. ${ }^{5}$

\footnotetext{
5 Dürrenmatt, Turmbau, 228.
} 
Eine ähnliche Reflexion hat vielleicht dazu geführt, dass in der französischen reformierten Kirche ein Glaubensbekenntnis ausformuliert wurde, das bei allen Grundaussagen des Glaubens ein »Trotz» einfügt:

"Wir glauben an Gott!

Trotz seines Schweigens und seines Geheimnisses

glauben wir, dass er lebendig ist.

Trotz des Übels und des Leidens

glauben wir, dass er die Welt für das Glück des Lebens erschaf-

fen hat

Trotz der Grenzen unserer Vernunft und der Widerstände

unseres Herzens

glauben wir an Gott.

Wir glauben an Jesus Christus!

Trotz der Jahrhunderte, die uns von der Zeit trennen, in der er gekommen ist,

glauben wir an sein Wort.

Trotz unseres Unverständnisses und unserer Ablehnung

glauben wir an seine Auferstehung.

Trotz seiner Schwäche und seiner Armut

glauben wir an sein Reich.

Wir glauben an den heiligen Geist!

Trotz allen Anscheins

glauben wir, dass er die Kirche führt.

Trotz des Todes

glauben wir an das ewige Leben.

Trotz des Unwissens und des Unglaubens

glauben wir, dass das Reich Gottes allen verheissen ist.

Amen. ${ }^{6}$

Wie bei Gianni Vattimo für die heutige Zeit "schwaches Denken" privilegiert wird, so könnte man auch die Glaubensgewissheit als eine "schwache Gewissheit» verstehen, die dem Zweifeln offen be-

\footnotetext{
6 Zitiert nach: H. Mottu (Hg., in Zusammenarbeit mit J. Cottin, D. Halter u. F. Moser), Confessions de foi réformées contemporaines. Et quelques autres textes de sensibilité protestante, Genf 2000, 157 (übers. P. Bühler).
} 
gegnet. Das hat die französische Schriftstellerin Sylvie Germain als die "Zerbrechlichkeit des Glaubens" angesprochen:

"Der Glaube muss, um die Prüfung der Zeit zu ertragen, um durchzuhalten und zu wachsen, einen doppelten Empfang vollziehen: seiner eigenen Zerbrechlichkeit, verbunden mit der conditio humana, und der Zerbrechlichkeit Gottes, der von allem entledigt ist ausser seinem Sein, dessen reinen Lebensgeschmack er allen anbietet. Diese zwei Zerbrechlichkeiten müssen miteinander in einen Dialog kommen. Aus ihrem Bund entsteht die Freude, die breite, lebhafte Freude, und das Licht.»

Der Zweifel steht ein für die Zerbrechlichkeit des Glaubens, gegen alle Absicherungen und Fixierungen. Er ist Einübung der schwachen Gewissheit, etwa mit Hilfe des Zweifels. In diesem Sinne gilt dem matthäischen Kleinglauben ein entschiedenes Lob.

- Dr. Pierre Bühler ist Professor für Systematische Theologie, insbes. Hermeneutik und Fundamentaltheologie am Institut für Hermeneutik und Religionsphilosophie an der Universität Zürich.

7 S. Germain, Fragilité de la foi, in: Quatre actes de présence, Paris, 2011, 63-79 (Zitat S. 78 ; übers. P. Bü.). 